\title{
Affections bucco-dentaires chez les personnes vivants avec le VIH/SIDA dans le district sanitaire de Koro, Mali
}

\section{Oral diseases in people living with HIV / AIDS in Koro health district, Mali}

\author{
KANE A.S.T. ${ }^{1}$, DIAWARA O.$^{2,3}$, SANGHO O. ${ }^{3}$, DIALLO B..$^{2,3}$, SITA-CRESP B. L.A. ${ }^{2}$, SAGARA I. ${ }^{3}$, TOURE K. ${ }^{2}$, COULIBALY B. ${ }^{2}$, BA A. ${ }^{2}$, BA \\ $\mathrm{M}^{2}{ }^{2}$ NIANG A. ${ }^{2}$, KAYENTAO K ${ }^{3}$, BA B. ${ }^{2}$, TRAORE $\mathrm{H}^{2}{ }^{2}$
}

\author{
1 Service Odontologie, Infirmerie Hôpital Militaire de Bamako \\ (IHB) MALI. \\ 2 Centre Hospitalier Universitaire Odontostomatologie de \\ Bamako (CHUOS) MALI. \\ ${ }^{3}$ DER de Santé Publique, Faculté de Médecine et \\ Odontostomatologie (FMOS) MALI. \\ Correspondance: Dr KANE Aboubacar Sidiki Thissé, \\ Service d'Odontologie, Infirmerie Hôpital Militaire de Bamako \\ IHB (Mali). Tel : 00223760272 83; BP : 2172 ; Bamako / \\ MALI. Email : aboukane3@yahoo.fr
}

\section{RESUME}

II s'agissait d'une étude transversale, descriptive portant sur les lésions bucco-dentaires chez les personnes vivant avec le $\mathrm{VIH} / \mathrm{SIDA}$ suivi dans le district sanitaire de Koro durant deux (2) mois. Cette étude portait sur 50 patients dont 31 femmes et 19 hommes. La majorité des patients venaient de l'aire du camp, 45 patients étaient mariés, $62 \%$ des cas étaient non alphabétisés, $96 \%$ avaient une sérologie de type I, $90 \%$ avaient des tartres et de plaques dentaires, 34\% avaient au moins 3 dents cariées. Les types de lésions buccales les plus fréquents étaient la candidose et les aphtes avec $24 \%$, les sièges les plus touches étaient le parodonte et la joue avec $24 \%$ et les besoins en soins bucco-dentaires étaient l'odontologie conservatrice endodontique avec $94 \%$ suivi de soins parodontaux (détartrages) avec $90 \%$. Les affections bucco-dentaires rencontrées chez les $\mathrm{PV} / \mathrm{VIH} / \mathrm{SIDA}$ étaient fréquentes et très variées. Ces affections bucco-dentaires survenaient à un stade avancé (stade II et III selon l'OMS), représentées en majeure partie par des candidoses surtout chez les patients aux traitements ARV sans les médicaments des infections opportunistes.

Mots-clés : Lésions buccales, personne vivants VIH/SIDA, Hôpital Militaire, Koro

\section{ABSTRACT}

This was a cross-sectional, descriptive study of oral lesions in people living with HIV / AIDS followed in the Koro health district for two (2) months. This study involved 50 patients, including 31 women and 19 men. The majority of patients were from the camp area, 45 were married, $62 \%$ were non-literate, $96 \%$ had type I serology, $90 \%$ had tartar and dental plaque, $34 \%$ had at least 3 decayed teeth. The most common types of oral lesions were candidiasis and canker sores with $24 \%$, the most affected seats were periodontium and cheek with $24 \%$ and oral care needs were endodontic conservative odontology with $94 \%$ followed. Periodontal care (scaling) with $90 \%$. Oral diseases encountered in HIV / AIDS were frequent and varied. These oral diseases occurred at an advanced stage (stage II and III according to WHO), represented for the most part by candidiasis, especially in patients with ARV treatment without the drugs of opportunistic infections.

Keywords: Oral lesions, person living HIV / AIDS, Military Hospital, Koro

\section{INTRODUCTION}

L'épidémie du VIH/SIDA demeure une priorité sanitaire, voir le développement à l'échelle mondiale. En fin 2010, on estimait à 34 millions le nombre de personnes vivant avec le VIH dans le monde, soit une hausse de $17 \%$ par rapport à 2001 [1].

À l'instar des autres pays du continent africain, le Mali est confronté à l'épidémie du VIH. Dans la population générale, la prévalence du VIH est estimée à 1,1\% selon l'Enquête Démographique et de Santé IV [2].

La cavité buccale est particulièrement susceptible au développement des lésions associées à l'infection au $\mathrm{VIH}$. Ces lésions sont nombreuses et variées d'où une sémiologie clinique qui est très riche [3]. Ces lésions sont parmi les premières manifestations à apparaître chez les personnes porteuses du virus [3].

Le VIH présente un certain nombre de défis au dentiste. Il est nécessaire de contrôler les risques d'infection et, malgré toutes les informations disponibles, une minorité de dentistes ont toujours des procédures de contrôle des infections incomplètes. II est également nécessaire de veiller à ce que le patient soit géré de manière optimale par le dentiste. II est importance d'établir des liens avec d'autres services pour gérer les demandes probables et les besoins des patients.

De nombreuses publications sur la connaissance, la pratique et les attitudes des prestataires de soins de santé bucco-dentaire se trouvent dans la littérature. La littérature indique une conformité accrue aux procédures de contrôle des infections. Alors que certains prestataires de soins de santé bucco-dentaire signalent des attitudes négatives à l'égard du traitement des patients atteints du $\mathrm{VIH} / \mathrm{SIDA}$, leur peur diminue, probablement en raison de leur conformité accrue aux procédures de contrôle des infections et d'un accès accru aux soins aux personnes atteintes du VIH/SIDA [4].

Au Mali aucune étude d'envergure nationale n'a été menée pour mesurer l'ampleur de ce problème. L'intérêt réside surtout dans le fait que la fréquence et l'incidence des affections bucco-dentaires peuvent augmenter au cours du VIH/SIDA. Leur détection, leur prévention et leur prise en charge doivent être intégrées dans les pratiques quotidiennes. 
L'objectif de ce travail était de déterminer les caractéristiques sociodémographiques et la fréquence des lésions bucco-dentaires chez les personnes séropositives sous antirétroviraux suivis au Csref de Koro.

\section{METHODOLOGIE}

II s'agissait d'une étude transversale descriptive dont l'enquête s'est déroulée sur une période de quatre (4) mois du 10 Mars 2016 au 09 Juin 2016. La population d'étude était constituée de toutes les personnes vivant avec VIH/SIDA (PVVIH) reçues dans le service pour le suivi et ayant accepté de répondre au questionnaire et d'être examinée. Une fiche d'enquête a été utilisée pour la collecte des données à partir des dossiers de suivi et à partir de l'observation des lésions bucco-dentaires. Les variables sociodémographiques et cliniques ont été récoltées. L'indice CAO a été déterminé. II s'agit d'un indice imaginé par Klein et Palmer depuis 1940, et qui permet de compter le nombre de dents adultes Cariées, Absentes et Obturées. Les données ont été saisies, traitées et analysées par le logiciel Epi-info version 3.5.3. Dans les considérations éthiques et déontologiques, il a été expliqué à chaque participant le but, les objectifs et la méthodologie de l'étude. Le consentement éclairé, volontaire et libre était obtenu avant l'enquête. Aucune donnée récoltée ne permet d'identifier un enquêté. La confidentialité a été garantie. L'étude a bénéficié de l'autorisation des autorités sanitaires.

\section{RESULTATS}

Nous avons colligé 50 patients. Le tableau I représente les caractéristiques sociodémographiques des enquêtés. Le sexe féminin était plus nombreux avec $62 \%$ avec un sex ratio de 0,6. La tranche d'âge de 30 à 39 ans était la plus touchée avec 20 cas soit $40 \%$. Les personnes mariées étaient les plus representées avec $90 \%$ des cas.

La plus part de nos patients avait le VIH type 1 soit $96 \%$. Seulement deux (4\%) avaient l'association type 1 et 2 . II y avait un cas de co-infection tuberculeuse. Au total, $90 \%$ des PVVIH/SIDA avaient des tartres et plaques dentaires. L'indice CAO est de 1,14 avec 40 dents cariées et 17 dents absentes. Les lésions buccales étaient présentes chez $86 \%$ des PVVI/SIDA. Le tableau II présente les types de lésions buccales, dominées par l'association candidoses et aphtes avec $24 \%$ des cas. L'évaluation a permis d'identifier des besoins en soins bucco-dentaires parodontaux chez $90 \%$ des patients, de Prothèses, d'extractions et d'endodontiques ou conservateurs respectivement chez $60 \%, 50 \%$ et $94 \%$.

La relation entre la présence de lésions des muqueuses buccales et la classification des stades selon l'OMS a montré que le stade I était dominant avec 24 cas soit $77,5 \%$ (tableau III). Les lésions étaient beaucoup plus fréquentes chez les PVVIH/SIDA qui avaient un taux de CD4 compris entre 200-499/mm ${ }^{3}$ (tableau VI).

Les PVVIH/SIDA, qui étaient sous anti rétro viraux (ARV) durant 9 mois et plus étaient les plus concernés par les lésions buccales avec 38 cas soit $86,4 \%$ (tableau $\mathrm{VI}$ ). Au total, $91,5 \%$ (32 personnes) des PVVIH/SIDA qui se brossaient une seule fois avaient des lésions buccales (tableau VI).

\section{DISCUSSION}

Dans notre étude, le sexe féminin était le plus représenté avec $62 \%$ soit un sex-ratio de 0,61 , ce résultat est comparable à celui de Soares GB et al. [5] qui trouve $53,1 \%$ et à celui d'autres auteurs [3,4].

La tranche d'âge la plus dominée était de 30 à 39 ans avec $40 \%$. Ce résultat est comparable aux travaux de BAH B. et al., Sacko A $[3,10]$ et à celui de Soares GB et al. [5]. Cette couche constitue la couche sociale la plus active et utile pour le développement économique de nos pays. Cependant certains auteurs comme Atessa $P$ et al en Iran et Sandeep Kumar et al. en Inde ont rapporté une prédominance masculine respectivement dans $82,7 \%$ et $60,3 \%[11,12]$. Par rapport à la situation matrimoniale, les personnes mariées étaient les plus nombreuses avec $90 \%$ suivie des célibataires et veuves avec $4 \%$ respectivement.

Pour les professions ou occupations, les femmes au foyer (ménagères) venaient en première position avec $25 \%$. La prédominance des femmes au foyer (ménagères) est également observée dans les études menées par d'autres auteurs $[4,6]$. Ce résultat pourrait s'expliquer par la situation socioculturelle (Koro une ville des dogons (ethnie) qui suivent jusqu'à présent leurs traditions par rapport à certaines pratiques comme la non scolarisation des filles).

Le VIH de type I dominait largement avec $96 \%$. Ce résultat est semblable à celui de BANCOLE $P$. $S$. et al. [4] au Bénin qui ont trouvés plus de $97,0 \%$ des PVVIH de type 1 et $45,0 \%$ selon la classification de l'OMS.

A l'examen endo-buccal, $90 \%$ des PVVIH/SIDA avaient des tartres sur les dents, l'état bucco-dentaire était de qualité moyenne avec $58 \%$ cas $34 \%$ avaient une mobilité dentaire, les dents cariées représentaient $34 \%$ des cas et les $30 \%$ ont eu au moins une dent absente, aucune dent n'a été traitée. Ce résultat pourrait s'expliquer par l'absence de cabinet dentaire au centre.

Par rapport aux lésions buccales, $86 \%$ avaient des lésions, les plus dominantes étaient la candidose et les aphtes avec $24 \%$ et les sièges des affections étaient le parodonte et les joues. Atessa $P$ et al. en Iran ont rapporté une parodontite sévère $(30 \%)$, la candidose pseudomembraneuse $(26 \%)$, la langue velue $(26 \%)$, l'hyperpigmentation mélanique $(20 \%)$ et la xérostomie $(16,7 \%)$ [11]. Ulrich $\mathrm{F}$ et al ont rapporté que $73,2 \%$ des participants examinés de leur étude présentaient une parodontite modérée à sévère [13].

Tandis que dans l'étude de Lydia M L une prévalence de la maladie parodontale de $72 \%$ a été trouvée (Près de $50 \%$ des patients avaient une parodontite chronique généralisée) [14]

Les besoins en soins bucco-dentaires conservateurs étaient de $94 \%$, et respectivement $60 \%$ et $90 \%$ pour la 
prise en charge parodontales et pour les restaurations prothétiques.

Le diagnostic précoce va permettre la mise en œuvre rapide de moyens thérapeutiques adaptés et peut, pour certaines lésions, mettre en évidence l'évolution vers les stades les plus avancés de l'infection.

\section{CONCLUSION}

Les affections bucco-dentaires rencontrées chez les $\mathrm{PV} / \mathrm{VIH} / \mathrm{SIDA}$ étaient fréquentes et très variées. Ces affections bucco-dentaires survenaient à un stade avancé (stade II et III selon l'OMS), représentées en majeure partie par des candidoses surtout chez les patients aux traitements ARV sans les médicaments des infections opportunistes.

Par ailleurs, la connaissance des relations existant entre l'apparition de certaines lésions buccales et l'évolution de la maladie est essentielle. La multiplication des études et une méta-analyse permettraient de mieux prédire l'évolution de la maladie en fonction de l'apparition des différentes lésions buccales.

\section{Déclaration de conflit d'intérêt}

Les auteurs déclarent n'avoir aucun conflit d'intérêt dans cette étude.

\section{REFERENCES}

1- Onu/Sida. Rapport Sur L'épidémie Mondiale Du Vih/Sida 2010. 2011, Mis à jour le 04/07/2012 disponible sur www.unaids.org

2-Ministère De La Santé Du Mali, Politique et protocole national de prise en charge ARV du VIH/SIDA. Juin 2010. 83p.

3-Bah B., Konate I., Goita D., Mbento G. A., Coulibaly A., Keita K., Affections Bucco-dentaires associées à l'infection à VIH dans le service de maladies infectieuses du CHU de Point-G, Bamako, Med Buccale Chir Buccale $2017 ; 23: 5-11$

4-Bancole P.S.A.; Biotchane I.; Zinsou R.; Yehouenou L.; Zannou D. Manifestations stomatologiques et infection à VIH au Centre national hospitalier et universitaire de Cotonou (Bénin) Med Buccale Chir. Buccale 2013;19:149-154.

5-Soares Gb, Garbin Ca, Rovida Ta, Garbin Aj, Oral health associated with quality

of life of people living with HIVIAIDS in Brazil.

Health Qual Life Outcomes. 2014: 1; $12-28$.

6-Hidreau P., L'épidémie du VIH/SIDA et sa situation dans un pays en voie de développement : le Bénin. Thèse d'exercice : Pharmacie : Nantes : $2006 ; 101$.

7-Gasparin Ab, Ferreira Fv, Danesi Cc, MendozaSassi Ra, Silveira J, Martinez Am, Zhang L, Cesar Ja., Prevalence of oral lesions in persons with HIV and associated factors in a southern Brazilian city Cad Saude Publica. 2009; 25(6):1307

8- Daniel Sj., Compliance with infection-control procedures and attitudes of oral health care providers toward patients with HIVIAIDS: a synthesis of the literature. J Dent Hyg. 1998;72 (3):33-45.
9- Green J., Psychological aspects of infection control and the care of the patient with HIV in dentistry.

Oral Dis. 1997;3,1:S225-8.

10. Sacko A. Etat parodontal des personnes vivant avec le VIH/SIDA suivis à l'hôpital de Sikasso et au Centre de Référence Kédougou Solidarité à propos de 124 cas. Thèse Chir Dent FMOS, Bamako 2016, 15, 56-78.

11. Atessa Pakfetrat Et Al. Manifestations buccales de patients infectés par le virus de l'immunodéficience humaine. Iran J Otorhinolaryngol . 2015; 27 (78): 43-54.

12. Sandeep Kumar, Prashant Mishra, Shilpa

Warhekar, Bhuvnesh Airen, Deepika Jain. État de santé bucco-dentaire et lésions oromuqueuses chez des patients vivant avec le VIH / sida en Inde: une étude comparative AIDS. ResTreat. 2014 : 480247.

13. Ulrich Fricke, Werner Geurtsen, Ingmar Staufenbiel ,Alexander Rahman, État parodontal des patients infectés par le VIH qui suivent un traitement antirétroviral par rapport aux patients naiffs de traitement par le VIH: une étude cas-témoin. Eur J Med Res . 2012; 17 (1): 2.

14. Lydia M Lopez, Maria Elena Guerra, Expérience de carie et statut parodontal pendant la grossesse dans un groupe de femmes enceintes infectées par le $\mathrm{VIH}+$ à Porto Rico.

J AIDS Clin Res. 2015; 6 (3): 434.

Liste des tableaux :

\begin{tabular}{|c|c|}
\hline $\begin{array}{l}\text { Tableau } \\
\text { sociodémograpl }\end{array}$ & Les \\
\hline caractéristiques & $n(\%)$ \\
\hline \multicolumn{2}{|l|}{ Sexe } \\
\hline$F$ & $31(62)$ \\
\hline M & $19(38)$ \\
\hline
\end{tabular}

Tranches d'âges

\begin{tabular}{lc} 
11-29 ans & $8(16)$ \\
$30-39$ ans & $20(40)$ \\
$40-49$ ans & $15(30)$ \\
$50-59$ ans & $7(14)$ \\
Situation matrimoniale & \\
Célibataire & $2(4)$ \\
Divorce & $1(2)$ \\
Marié(e) & $45(90)$ \\
veuf/veuve & $2(4)$ \\
Total & $50(100)$ \\
\hline
\end{tabular}

Tableau II : Répartition des patients en fonction des types de lésion

\begin{tabular}{ll}
\hline \hline Types de lésions & $\mathbf{n}(\%)$ \\
\hline Aphte & $7(14)$ \\
Autres & $7(14)$ \\
\hline
\end{tabular}




\begin{tabular}{lc}
\hline Candidoses & $9(18)$ \\
Candidoses + aphtes & $12(24)$ \\
Gingivite & $1(2)$ \\
GUN & $2(4)$ \\
Parodontite chronique & $10(20)$ \\
PUN & $2(4)$ \\
Total & $\mathbf{5 0 ( 1 0 0 )}$ \\
\hline
\end{tabular}

Tableau III : Analyse bivariée entre la présence des lésions et certaines variables indépendantes

\begin{tabular}{lcc}
\hline Variables & Oui $\mathrm{n}(\%)$ & Total \\
\hline Stades de classification & & \\
selon I'OMS & & \\
Stade I & $24(77,5)$ & 31 \\
Stade II & $14(100)$ & 1 \\
Stade III & $4(100)$ & 4 \\
Stade IV & $1(100)$ & 1 \\
\hline
\end{tabular}

\begin{tabular}{lcc} 
Total & $\mathbf{4 3 ( 8 6 )}$ & $\mathbf{5 0}$ \\
Taux de TCD4 & & \\
$>500 / \mathrm{mm} 3$ & $6(85,8)$ & 7 \\
$200-499 / \mathrm{mm} 3$ & $25(86,3)$ & 29 \\
$<200 / \mathrm{mm} 3$ & $4(100)$ & 4 \\
ND & $8(80)$ & 10 \\
Total & $43(86)$ & 50 \\
Durée sous ARV & & \\
$1-3$ mois & $2(100)$ & 2 \\
$6-9$ mois & $3(75)$ & 4 \\
9 mois et plus & $38(86,4)$ & 44 \\
Total & $43(86)$ & 50 \\
Fréquence de brossage & & \\
2 fois & $5(55,6)$ & 9 \\
3fois & $1(100)$ & 1 \\
Ne sait pas & $5(100)$ & 5 \\
Une fois & $32(91,5)$ & 35 \\
Total & $43(86)$ & 50 \\
\hline
\end{tabular}

